\title{
PERspective on PER phosphorylation
}

\author{
Justin Blau' ${ }^{1}$ \\ Department of Biology, New York University, New York, New York 10003, USA
}

Period (PER) proteins are essential parts of the molecular clocks that control circadian rhythms in flies and mammals. Phosphorylation regulates PER's stability and subcellular localization; however, the physiologically relevant sites have been difficult to identify in spite of knowing the relevant kinase. In this issue of Genes \& Development, Chiu and colleagues (pp. 1758-1772) identify a key phosphorylation site on PER that recruits the F-box protein Slimb to trigger PER degradation and set clock speed.

In the beginning there was per. Or at least three mutant alleles of the Drosophila period gene: a null mutation $\left(\right.$ per $\left.^{O}\right)$ that made flies behaviorally arrhythmic, and two period-altering alleles (per ${ }^{\text {Short }}$ and per $^{\text {Long }}$ ) that, respectively, gave flies 19-h and 29-h rhythms in constant darkness (Konopka and Benzer 1971). These mutants were surprising to many when described in 1971 since there were doubts that animal behavior (in this case, the $\sim 24-\mathrm{h}$ circadian rest/activity cycles of fruitflies) would be so dependent on a single gene. Humans and other mammals have three Per genes, which suggests some redundancy. However, humans heterozygous for a point mutation in hPer2 that causes a single amino acid change suffer from Familial Advanced Sleep Phase Syndrome, and their sleep/wake cycles run fast, repeating every $23 \mathrm{~h}$ (Jones et al. 1999; Toh et al. 2001). Thus, single gene mutations can profoundly affect circadian behavior.

per is one component of the internal clocks that allow flies and mammals to anticipate regular daily environmental changes and to maintain $\sim 24-\mathrm{h}$ rhythms of behavior even in constant darkness. In addition to behavioral rhythms, mammals also have metabolic circadian rhythms-for example, daily core body temperature changes and gating of the cell cycle. Disruptions to the circadian system are obvious in jetlag, and have also been associated with depression and increased incidence of cancer (for review, see Hastings et al. 2003).

Here I briefly describe the fly clock, although the mammalian clock works in a similar manner and uses related genes (for more details, see Hardin 2005). First,

[Keywords: Circadian rhythms; Drosophila; PER; $\beta$-TrCP/SLIMB; CK $1 \varepsilon /$ DBT; F-box protein; phosphorylation]

${ }^{1}$ Correspondence.

E-MAIL justin.blau@nyu.edu; FAX (212) 995-4015.

Article is online at http://www.genesdev.org/cgi/doi/10.1101/gad.1696408. per transcription is activated by the CLOCK (CLK) and CYCLE (CYC) transcription factors. per mRNA accumulates, but the initially translated PER is unstable, leading to a substantial lag in the peak of PER protein compared with per RNA. Cytoplasmic PER accumulation requires binding to TIMELESS (TIM), the protein product of another direct CLK/CYC target and, like per, a rhythmically expressed gene. PER and TIM then enter the nucleus in a time-gated manner. In the nucleus, PER represses further expression of per, tim, and many other CLK/CYC direct targets by removing CLK and CYC from DNA. Negative feedback ends with PER degradation, allowing CLK/CYC to reactivate transcription of per and tim, and a new cycle begins. In this way, per RNA and PER protein levels oscillate taking $24 \mathrm{~h}$ for one complete cycle, paralleling flies' behavioral rhythms.

PER is progressively phosphorylated with time over the course of the night and early morning and is then degraded (Edery et al. 1994). Mutations in double-time $(d b t)$, which encodes a CK1 family member, alter rates of PER phosphorylation. In turn, this changes the timing of molecular clocks and the resulting behavior, suggesting that DBT regulates PER phosphorylation and stability (Price et al. 1998). Subsequently, it was shown that PER and DBT stably associate in flies (Kloss et al. 2001). However, the precise sites on PER that are phosphorylated by DBT have proved elusive, for several reasons: the large number of serine and threonine residues in PER (>250) and the inactivity of bacterially produced DBT, as well as the difficulty in obtaining enough PER from flies to map phosphorylation sites. In this issue of Genes \& Development, Chiu et al. (2008) report how they surmounted these problems by using a cell culture system to identify DBT-dependent phosphorylation sites on PER. They identify a key phosphorylation site at the $\mathrm{N}$ terminus of PER that helps recruit the F-box protein Slimb in vitro. Importantly, altering this one amino acid in PER in vivo lengthened behavioral rhythms by almost $7 \mathrm{~h}$, emphasizing its importance in setting clock speed.

\section{PER phosphorylation}

Many of the genes that regulate circadian timing alter PER phosphorylation and/or PER stability. Together, they have led to the idea that the rate of PER phosphorylation determines the speed of the clock. These relevant 


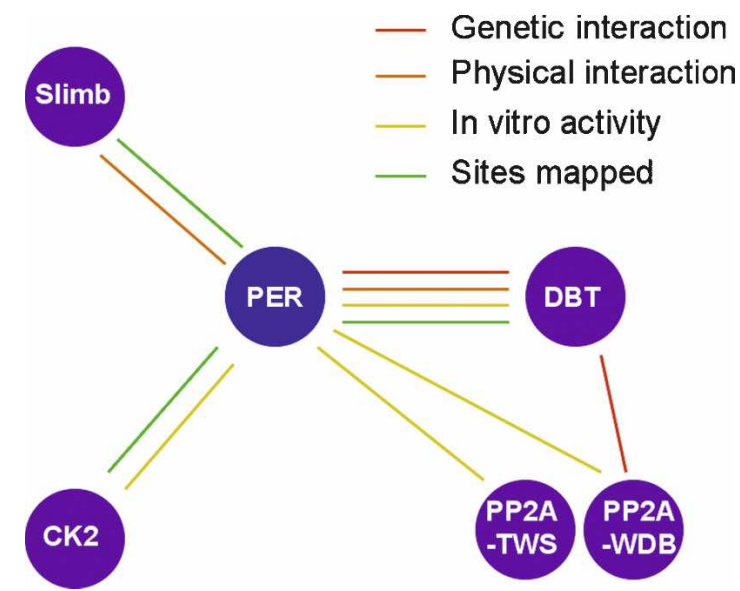

Figure 1. Network regulating PER protein phosphorylation and stability. All of the protein products shown alter circadian rhythms and alter PER phosphorylation and/or stability when mutated in flies. The connectors indicate additional support for the network coming from diverse lines of evidence. See the text for details. The study by Chiu et al. (2008) identified sites on PER phosphorylated by DBT and recognized by Slimb (green lines).

clock genes encode two kinases (DBT and CK2); two Protein Phosphatase 2A (PP2A) complexes that differ by having either Twins (Tws) or Widerborst (Wdb) as their associated regulatory subunit; and the Slimb F-box protein (for review, see Harms et al. 2004). This is shown as a mininetwork in Figure 1, with the different types of evidence supporting the network represented by different colors. Thus, there are genetic interactions between specific per and $d b t$ alleles (shown in red in Fig. 1; Rothenfluh et al. 2000) and physical interactions between PER and DBT demonstrated in vitro and in flies (shown in orange in Fig. 1; Kloss et al. 1998, 2001). PP2A can directly dephosphorylate PER in vitro (yellow), supporting that interaction, and there are also genetic interactions suggesting that PP2A-WDB opposes DBT function by dephosphorylating PER in the nucleus (red in Fig. 1; Sathyanarayanan et al. 2004). There is also biochemical evidence from flies that Slimb recognizes phosphorylated PER and targets it for degradation via the ubiquitin-proteasome pathway (Grima et al. 2002; Ko et al. 2002). Finally, CK2 can phosphorylate PER toward its N terminus in vitro, and mutation of these CK2 sites on PER in flies gives similar circadian phenotypes as reducing CK2 activity (Lin et al. 2005).

Nevertheless, despite the overwhelming array of evidence that DBT, PP2A, and Slimb together regulate PER stability and function, there are some surprisingly important details missing: On which residues is PER phosphorylated by DBT? And why is PER so heavily phosphorylated? Chiu et al. (2008) approached these questions first by identifying regions of PER that contribute to PER stability using a simple Drosophila cell culture assay with constitutive per and inducible $d b t$ expression. Having found that the $\mathrm{N}$-terminal 100 amino acids of PER are required for DBT-mediated PER destabiliza- tion, they identified Ser47 as having the largest effect of any single amino acid in that region. Mutation of Ser47 to a nonphosphorylatable alanine stabilized PER and potently reduced PER binding to its F-box, Slimb. Conversely, mutating Ser47 to the phospho-mimetic aspartic acid destabilized PER and dramatically increased interaction with Slimb.

Is the phosphorylation of Ser47 as important in vivo as it is in vitro? Three lines of evidence lead to a resounding "Yes." First, Chiu et al. (2008) show that the same mutation that increased PER stability in cell culture (S47A) lengthens adult locomotor rhythms from $24 \mathrm{~h}$ to $31 \mathrm{~h}$ (Fig. 2). Second, they show that the mutation that decreased PER stability (S47D) shortens the behavioral cycle to $22 \mathrm{~h}$ (Fig. 2). And third, antibodies specific for phospho-Ser47 show that this residue is, indeed, phosphorylated in flies. Finally, Chiu et al. (2008) went on to show that in vitro purified DBT can directly phosphorylate Ser47. The only piece missing would be to test if Ser47 is unphosphorylated in flies lacking DBT function. However, $d b t$ is an essential gene, making this experiment hard to perform.

Although DBT is stably bound to PER, the phosphospecific antibody experiments indicate that phosphorylation of Ser47 is a relatively late event in the life of a PER protein. This makes sense since once Ser47 is phosphorylated, it is only a matter of time before Slimb is recruited and PER is degraded. So to allow PER to endure for many hours, it seems that DBT phosphorylation of Ser47 is deliberately poor. Or, perhaps that PP2A-mediated dephosphorylation of Ser47 is very fast-or both. Similarly the Slimb-binding site surrounding Ser47 also seems "deliberately bad" - it is not a good Slimb consensus sequence, and again this may be important in stretching out the half-life of a PER protein to help fill a 24-h cycle.

\section{More PER phosphorylation}

Chiu et al. (2008) also used the cell culture system to identify additional PER phosphorylation sites via mass
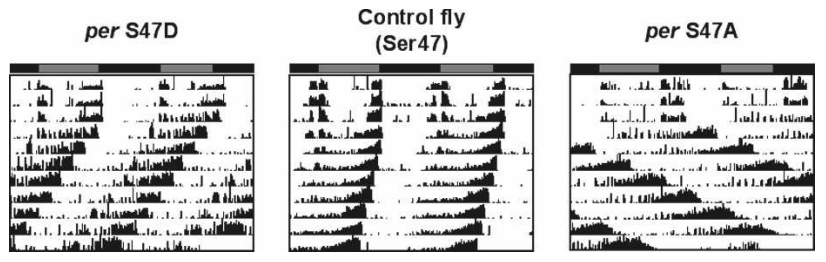

Figure 2. Dramatic circadian phenotypes from single amino acid changes in PER. The locomotor activity of single flies is shown for three PER variants: PER S47D (left), wild type PER (middle), and PER S47A (right). Flies were monitored in light:dark cycles for the first $3 \mathrm{~d}$ of the experiment, before transfer to constant darkness to reveal the free-running periods. per S47D flies have shorter than normal rhythms $(\sim 22 \mathrm{~h})$, compared with $\sim 23.5 \mathrm{~h}$ for control flies. per S47A flies have dramatically long rhythms $(\sim 31 \mathrm{~h})$. See the text for details. The data were kindly provided by Joanna Chiu. 
spectrometry. Among the 25 sites identified, the DBTmediated PER phosphorylation site at Ser589 stood out. Ser589 is the amino acid mutated in the original per short $^{\text {She }}$ allele identified by Konopka and Benzer (1971). By this point, you will no doubt be predicting that in per $^{\text {Short }}$, Ser589 is mutated to a phospho-mimetic Asp or Glu to speed up the clock just as Chiu et al. (2008) had done when engineering the S47D mutation to give 22-h rhythms. Unfortunately, this is not the case- $-p^{\text {Short }}$ has a Ser-to-Asn change at position 589. Why, then, is per $^{\text {Short }}$ short and not long? Clearly not all phosphorylation events on PER have the same effect. While phosphorylation of Ser589 and the surrounding residues seems to normally slow down the clock, phosphorylation around Ser47 normally speeds up the clock. Similarly, the hPer2 mutation that underlies Familial Advanced Sleep Phase Syndrome is the loss of a phosphorylatable Ser that speeds up the clock (Toh et al. 2001), in an analogous manner to the per ${ }^{\text {Short }}$ mutation.

The phospo-mapping by Chiu et al. (2008) for Drosophila PER and by Vanselow et al. (2006) for mPER2 indicates that PER phosphorylation occurs in discrete clusters. Together, they provide excellent support for the idea that PER proteins have different domains whose phosphorylation has different effects. Some domains could affect PER stability in the nucleus and others in the cytoplasm. Other regions of PER potentially regulated by phosphorylation include domains that help time nuclear entry (Meyer et al. 2006) and that repress transcription (Chang and Reppert 2003). These different clusters could then regulate different aspects of PER function-even if phosphorylated by the same kinase (DBT/ CK1 $\varepsilon$ in flies and mammals, respectively). This idea helps to explain some confusing phenotypes that arise from mutations that alter DBT/CK1 $\varepsilon$ activity since the mutant enzymes seem to differentially affect phosphorylation at different domains of PER (Gallego et al. 2006; Xu et al. 2007; Meng et al. 2008).

Indeed, it is striking that Chiu et al. (2008) show that altering Ser47 stabilizes PER but PER still becomes hyperphosphorylated. Until relatively recently, the dogma was that PER phosphorylation simply preceded degradation. Now it is clear that PER proteins are phosphorylated in numerous domains to regulate PER activity and help PER accurately track time. Elucidation of the function of these different PER domains and their regulation by phosphorylation now seems tractable.

\section{PERspective: transcriptional or post-translational regulation?}

Last year, the circadian rhythms field was invited to share its ideas at the Cold Spring Harbor Symposium for the first time since 1960. One of the questions discussed was whether transcriptional regulation or post-transcriptional regulation is more important in the clock. A case can be made for each side. For example, one could argue that post-transcriptional regulation is more important since the most dramatic period length changes in circadian mutants tend to affect post-translational regulation (e.g., Smith et al. 2008 and the study from Chiu et al. 2008). However, the animal clock transcriptional regulatory loops are so intertwined (for review, see Hardin 2005) that mutations in clock transcription factors may be buffered by the connectivity of the system as long as they allow sufficient gene expression to take place. Again supporting the post-translational camp, Yang and Sehgal (2001) found that constitutive expression of per and tim could rescue the rhythmic behavior of per $^{01}$; $t \mathrm{~m}^{01}$ double null mutants. However, a recent reanalysis of this experiment indicates that the extent of rhythmicity was probably overestimated and most of the rhythms detected were weak (Hall et al. 2007). However the strongest piece of evidence for a post-translational clock comes from Cyanobacteria, where circadian rhythms in phosphorylation of the KaiC clock protein can be seen for several days in a test tube when mixing three proteins together-with no DNA or RNA present (Nakajima et al. 2005). Thus a purely post-translational clock can function. However, a subsequent study revealed that robust and accurate in vivo circadian rhythms of Cyanobacteria require transcriptional feedback and can even proceed without rhythmic KaiC phosphorylation (Kitayama et al. 2008).

So is transcriptional or post-transcriptional regulation more important? I believe the answer is "No." Instead, it seems that each tier of regulation adds to the robustness and accuracy of these internal molecular clocks that help animals stay synchronized with their environment and keep their internal organs working together in harmony.

\section{Acknowledgments}

I thank David Dahdal for comments on the manuscript. Work in my laboratory is funded by NIH grant GM063911.

\section{References}

Chang, D.C. and Reppert, S.M. 2003. A novel C-terminal domain of Drosophila PERIOD inhibits dCLOCK:CYCLE-mediated transcription. Curr. Biol. 13: 758-762.

Chiu, J.C., Vanselow, J.T., Kramer, A., and Edery, I. 2008. The phospho-occupancy of an atypical SLIMB binding site on PERIOD that is phosphorylated by DOUBLETIME controls the pace of the clock. Genes \& Dev. (this issue) doi: 10.1101/ gad.1682708.

Edery, I., Zwiebel, L.J., Dembinska, M.E., and Rosbash, M. 1994. Temporal phosphorylation of the Drosophila Period protein. Proc. Natl. Acad. Sci. 91: 2260-2264.

Gallego, M., Eide, E.J., Woolf, M.F., Virshup, D.M., and Forger, D.B. 2006. An opposite role for tau in circadian rhythms revealed by mathematical modeling. Proc. Natl. Acad. Sci. 103: $10618-10623$.

Grima, B., Lamouroux, A., Chelot, E., Papin, C., LimbourgBouchon, B., and Rouyer, F. 2002. The F-box protein Slimb controls the levels of clock proteins Period and Timeless. Nature 420: 178-182.

Hall, J.C., Chang, D.C., and Dolezelova, E. 2007. Principles and problems revolving around rhythm-related genetic variants. Cold Spring Harb. Symp. Quant. Biol. 72: 215-232.

Hardin, P.E. 2005. The circadian timekeeping system of Dro- 
sophila. Curr. Biol. 15: R714-R722. doi: 10.1016/j.cub.2005 08.019 .

Harms, E., Kivimae, S., Young, M.W., and Saez, L. 2004. Posttranscriptional and posttranslational regulation of clock genes. J. Biol. Rhythms 19: 361-373.

Hastings, M.H., Reddy, A.B., and Maywood, E.S. 2003. A clockwork web: Circadian timing in brain and periphery, in health and disease. Nat. Rev. Neurosci. 4: 649-661.

Jones, C.R., Campbell, S.S., Zone, S.E., Cooper, F., DeSano, A., Murphy, P.J., Jones, B., Czajkowski, L., and Ptcek, L. 1999. Familial advanced sleep-phase syndrome: A short-period circadian rhythm variant in humans. Nat. Med. 5: 1062-1065.

Kitayama, Y., Nishiwaki, T., Terauchi, K., and Kondo, T. 2008. Dual KaiC-based oscillations constitute the circadian system of Cyanobacteria. Genes \& Dev. 22: 1513-1521.

Kloss, B., Price, J.L., Saez, L., Blau, J., Rothenfluh, A., Wesley, C.S., and Young, M.W. 1998. The Drosophila clock gene double-time encodes a protein closely related to human casein kinase I $\varepsilon$. Cell 94: 97-107.

Kloss, B., Rothenfluh, A., Young, M.W., and Saez, L. 2001. Phosphorylation of PERIOD is influenced by cycling physical associations of DOUBLE-TIME, PERIOD, and TIMELESS in the Drosophila clock. Neuron 30: 699-706.

Ko, H.W., Jiang, J., and Edery, I. 2002. Role for Slimb in the degradation of Drosophila Period protein phosphorylated by Doubletime. Nature 420: 673-678.

Konopka, R.J. and Benzer, S. 1971. Clock mutants of Drosophila melanogaster. Proc. Natl. Acad. Sci. 68: 2112-2116.

Lin, J.M., Schroeder, A., and Allada, R. 2005. In vivo circadian function of casein kinase 2 phosphorylation sites in Drosophila PERIOD. J. Neurosci. 25: 11175-11183.

Meng, Q.J., Logunova, L., Maywood, E.S., Gallego, M., Lebiecki, J., Brown, T.M., Sladek, M., Semikhodskii, A.S., Glossop, N.R., Piggins, H.D., et al. 2008. Setting clock speed in mammals: The CK1 $\varepsilon$ tau mutation in mice accelerates circadian pacemakers by selectively destabilizing PERIOD proteins. Neuron 58: 78-88.

Meyer, P., Saez, L., and Young, M.W. 2006. PER-TIM interactions in living Drosophila cells: An interval timer for the circadian clock. Science 311: 226-229.

Nakajima, M., Imai, K., Ito, H., Nishiwaki, T., Murayama, Y., Iwasaki, H., Oyama, T., and Kondo, T. 2005. Reconstitution of circadian oscillation of cyanobacterial KaiC phosphorylation in vitro. Science 308: 414-415.

Price, J.L., Blau, J., Rothenfluh, A., Abodeely, M., Kloss, B., and Young, M.W. 1998. double-time is a novel Drosophila clock gene that regulates PERIOD protein accumulation. Cell 94: 83-95.

Rothenfluh, A., Abodeely, M., and Young, M.W. 2000. Shortperiod mutations of per affect a double-time-dependent step in the Drosophila circadian clock. Curr. Biol. 10: 1399-1402.

Sathyanarayanan, S., Zheng, X., Xiao, R., and Sehgal, A. 2004. Posttranslational regulation of Drosophila PERIOD protein by protein phosphatase 2A. Cell 116: 603-615.

Smith, E.M., Lin, J.M., Meissner, R.A., and Allada, R. 2008. Dominant-negative $\mathrm{CK} 2 \alpha$ induces potent effects on circadian rhythmicity. PLoS Genet. 4: 99-109.

Toh, K.L., Jones, C., He, Y., Eide, E.J., Hinz, W.A., Virshup, D.M., Ptacek, L.J., and Fu, Y.H. 2001. An hPer2 phosphorylation site mutation in familial advanced sleep phase syndrome. Science 291: 1040-1043.

Vanselow, K., Vanselow, J.T., Westermark, P.O., Reischl, S., Maier, B., Korte, T., Herrmann, A., Herzel, H., Schlosser, A., and Kramer, A. 2006. Differential effects of PER2 phosphorylation: Molecular basis for the human familial advanced sleep phase syndrome (FASPS). Genes \& Dev. 20: 2660-2672.
Xu, Y., Toh, K.L., Jones, C.R., Shin, J.Y., Fu, Y.H., and Ptacek, L.J. 2007. Modeling of a human circadian mutation yields insights into clock regulation by PER2. Cell 128: 59-70.

Yang, Z. and Sehgal, A. 2001. Role of molecular oscillations in generating behavioral rhythms in Drosophila. Neuron 29: $453-467$. 


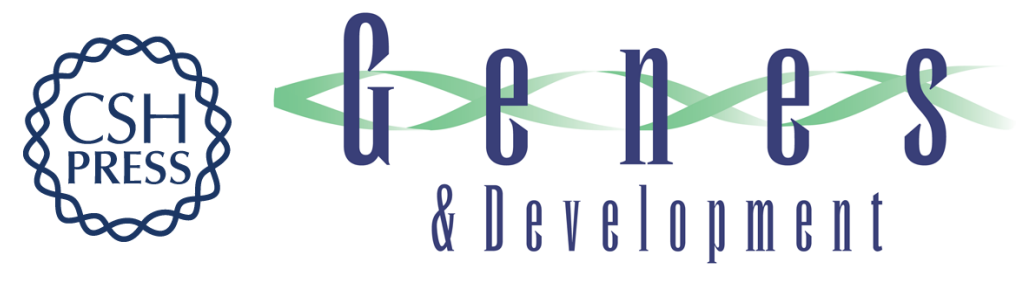

\section{PERspective on PER phosphorylation}

Justin Blau

Genes Dev. 2008, 22:

Access the most recent version at doi:10.1101/gad.1696408

Related Content The phospho-occupancy of an atypical SLIMB-binding site on PERIOD that is phosphorylated by DOUBLETIME controls the pace of the clock Joanna C. Chiu, Jens T. Vanselow, Achim Kramer, et al.

Genes Dev. July, 2008 22: 1758-1772

References This article cites 27 articles, 11 of which can be accessed free at:

http://genesdev.cshlp.org/content/22/13/1737.full.html\#ref-list-1

Articles cited in:

http://genesdev.cshlp.org/content/22/13/1737.full.html\#related-urls

License

Email Alerting Receive free email alerts when new articles cite this article - sign up in the box at the top

Service right corner of the article or click here.

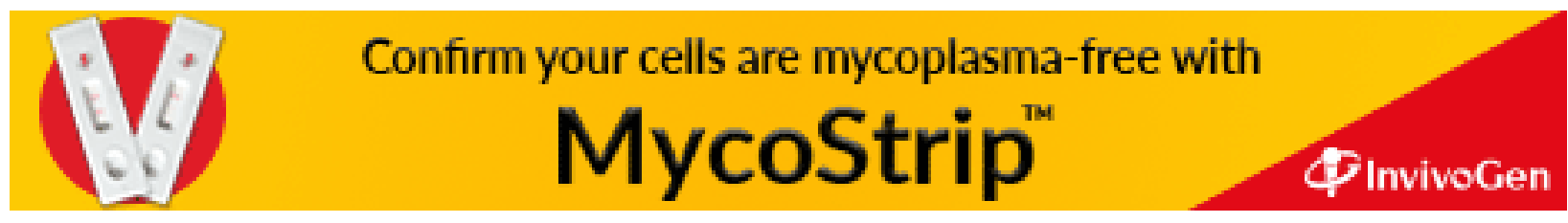

